\title{
Long non-coding RNA LINC00662 promotes proliferation and migration of breast cancer cells via regulating the miR-497-5p/ EgIN2 axis
}

\author{
Long Cheng ${ }^{1}$, Zihe Xing ${ }^{1}$, Peng Zhang ${ }^{1}$ and Wuqin $\mathrm{Xu}^{2}{ }^{\square}$ \\ 'Clinical Laboratory, The First Affiliated Hospital of Wannan Medical College, Wuhu City, Anhui Province, 241001, China; 2Department of Pathol- \\ ogy, The First Affiliated Hospital of Wannan Medical College, Wuhu City, Anhui Province, 241001, China
}

Previous reports indicated that ong intergenic non-protein coding RNA 662 (LINC00662) plays a crucial role in several human cancers. Here, we studied the expression pattern of LINC00662 and explored its function in human breast cancer. The expression level of LINC00662 was determined in human breast cancer cell lines and tissues by real-time quantitative polymerase chain reaction (RT-qPCR). Cytoplasmic and nuclear RNA from MDAMB-157 cells were extracted to analyze the subcellular location of LINC00662. Moreover, the MTT assay, woundhealing assay, colony-forming assay and transwell assay were employed in MDA-MB-157 cells to detect the effect of LINC00662 on cell apoptosis, invasion, migration and proliferation, respectively. LINC00662-specific miRNA and miRNA-gene axis were examined in a dual-luciferase reporter assay and Western blot. We found that LINC00662 was overexpressed in both breast cancer cell lines and tissue compared to normal breast cell lines and healthy breast tissue. Analysis of subcellular localization revealed that LINC00662 was mainly found in the cytoplasm. Furthermore, LINC00662 silencing reduced cell viability and inhibited the proliferation, migration and invasion of MDA-MB-157 cells. Bioinformatics analysis predicted that LNC00662 binds to miR-497-5p. A series of studies confirmed that LINC00662 directly interacted with miR-497-5p and downregulated its expression in MDA-MB-157 cells. MiR-497-5p knockdown significantly reversed the inhibitory effect of shLINC00662. Moreover, egl-9 family hypoxia inducible factor 2 (EgIN2) was verified as a target of miR-497-5p. Overall, our results demonstrated that overexpression of LINC00662 accelerated the malignant growth of breast cancer cells via sponging miR-497-5p and upregulating EgIN2 expression, and indicate that targeting LINC00662 may represent a novel strategy for breast cancer therapy.

Key words: LINC00662, miR-497-5p, EgIN2, human breast cancer Received: 10 March, 2020; revised: 25 March, 2020; accepted: 07 April, 2020; available on-line: 19 June, 2020

『e-mail: chenglong06@126.com

Acknowledgements of Financial Support: This work was supported by the Open Project of Key Laboratory of Non-coding RNA Transformation Research of Anhui Higher Education Institution (Wannan Medical College) (Grant no. RNA201904) and Anhui Province Key Laboratory of Active Biological Macro-molecules Research (Grant no. LAB201809).

Abbreviations: $\mathrm{BC}$, breast cancer; $\mathrm{ECL}$, enhanced chemiluminescence; EgIN2, egl-9 family hypoxia inducible factor 2; FBS, fetal bovine serum; GAPDH, glyceraldehyde-phosphate dehydrogenase; LINC00662, long intergenic non-protein coding RNA 662; IncRNAs, long non-coding RNAs; MUT, mutation; NC, negative control; PVDF, polyvinylidene fluoride; RTqPCR, real-time quantitative polymerase chain reaction; SDS-PAGE, sodium dodecylsulphate polyacrylamide gel electrophoresis; S.D., standard deviation; UTR, Untranslated Regions

\section{INTRODUCTION}

Breast cancer is the most common malignant tumor and the sixth leading cause of death from cancer among women around the world (Bray et al., 2018). In China, breast cancer ranks the third among female malignant tumors (Chen et al., 2016). In recent years, due to the continuous development of treatment technology and the popularization of cancer prevention measures, the incidence of breast cancer is on the decline. But globally, the number of new cases still continues to grow at a rate of about two million a year ( $\mathrm{Li}$ et al., 2012). In western developed countries, breast cancer is the most common malignant tumor in women. With the continuous progress of medicine, the 5-year survival of breast cancer patients can be improved with surgical treatment. However, the prognosis and long-term survival rate in breast cancer patients are still not ideal due to a variety of factors (Fasih et al., 2012). Therefore, it is urgently needed to develop an effective strategy to improve early detection of breast cancer. However, despite increasing reports on the molecular mechanisms of breast cancer development, still little is known about the exact molecular mechanisms of breast cancer pathogenesis.

Long non-coding RNAs (lncRNAs) belong to a wider group of RNAs that do not code proteins. The length of lncRNAs is over 200 base pairs, which differentiates them from the other non-coding RNAs (Qiu et al., 2013). During the past few decades, a great amount of reports has shown that lncRNAs can act as scaffolds or sponges to modulate gene expression (Lalevee \& Feil 2015), which allows them to play some vital roles in cell proliferation, differentiation or apoptosis (Xue et al., 2017; DiStefano 2018). Numerous studies (Lv et al., 2019; Sun et al., 2019) have provided clear evidence that the aberrant lncRNA played an oncogenic or tumor-suppressive roles in breast cancer and lncRNAs dysregulation could serve as a potential prognostic and diagnostic biomarker for breast cancer, as well as a potential therapeutic target. Long intergenic non-protein coding RNA 662 (LINC00662) was first identified in lung squamous cell carcinoma (Liu et al., 2017). Next, LINC00662 was observed in several other tumors, including lung cancer (Gong et al., 2018) and gastric cancer (Liu et al., 2018). The loss-of-function and gain-of-function strategies con- 
Table 1 Relationship between LINC00662 and clinico-pathological parameters

\begin{tabular}{|c|c|c|c|c|}
\hline \multirow{2}{*}{ Parameters } & \multirow{2}{*}{ Number of patients } & \multicolumn{3}{|c|}{ LINC00662 expression } \\
\hline & & Low (<median) & High ( $\geq$ median) & $P$ value \\
\hline Number & 47 & 23 & 24 & \\
\hline \multicolumn{5}{|l|}{ Age (years) } \\
\hline$\geq$ Mean (48) & 25 & 13 & 12 & \multirow{2}{*}{0.654} \\
\hline$<$ Mean (48) & 22 & 10 & 12 & \\
\hline \multicolumn{5}{|c|}{ Pathologic grade } \\
\hline 1 & 15 & 10 & 5 & \multirow{2}{*}{0.096} \\
\hline II-III & 32 & 13 & 19 & \\
\hline \multicolumn{5}{|l|}{ Clinical stage } \\
\hline I-II & 32 & 19 & 13 & \multirow{2}{*}{$0.037^{*}$} \\
\hline III & 15 & 4 & 11 & \\
\hline \multicolumn{5}{|c|}{ Lymph node status } \\
\hline Metastasis & 26 & 9 & 17 & \multirow{2}{*}{$0.029 *$} \\
\hline No metastasis & 21 & 14 & 7 & \\
\hline \multicolumn{5}{|l|}{ HER2 status } \\
\hline Positive & 26 & 13 & 13 & \multirow{2}{*}{0.871} \\
\hline Negative & 21 & 10 & 11 & \\
\hline \multicolumn{5}{|l|}{ ER status } \\
\hline Positive & 26 & 12 & 14 & \multirow{2}{*}{0.671} \\
\hline Negative & 21 & 11 & 10 & \\
\hline \multicolumn{5}{|l|}{ PR status } \\
\hline Positive & 23 & 10 & 13 & \multirow{2}{*}{0.464} \\
\hline Negative & 24 & 13 & 11 & \\
\hline
\end{tabular}

firmed the tumor-promoting roles of LINC00662 ( $\mathrm{Li}$ et al., 2019).

LINC00662 has been studied as biomarker in several kinds of human tumors, but its involvement in breast cancer is still unknown. In the present study, we found that LINC00662 may play an important role as a potential oncogene in breast cancer. First, we show the function of LINC00662 in breast cancer cells. Then, with a series of experiments we demonstrate that LINC00662 could upregulate EglN2 expression by sponging miR497-5p, indicating that LINC00662 may take part in the inactivation of the miR-497-5p-EglN2 pathway. Our results indicate $t$ lncRNA LINC00662 is a new potential biomarker for breast cancer.

\section{MATERIALS AND METHODS}

Tissue samples. The present study was approved by the Ethics Committee of The First Affiliated Hospital of Wannan Medical College. A total of 47 breast cancer samples and 20 adjacent normal tissue samples were obtained from the patients. All cases were validated by histological examination for breast cancer according to World Health Organization criteria. The tissue specimens were formalin fixed immediately after surgical excision from patients. For clinical information on patients, see Table 1.

Cell lines. Human breast cancer cell lines (MDAMB-468, MCF-7 and MDA-MB-157) and human breast fibroblast cell line (CCD-1095Sk) were purchased from American Type Culture Collection (ATCC, Manassas, VA, USA). MDA-MB-468 and MDA-MB-157 cells were cultured in Leibovitz's L-15 Medium (Gibco, MA, USA) supplemented with $10 \%$ fetal bovine serum (FBS, Gibco). MCF-7 and CCD-1095Sk cells were cultured in Minimum Essential Medium (MEM, Gibco) supplemented with 10\% FBS.

Nuclear and cytoplasmic fractionation. Cytoplasmic and Nuclear RNA Purification Kit (Norgen Biotek Corp. Thorold, Canada) were used to extract the cytoplasm and nuclear RNAs from MDA-MB-157 cells. After that, RT-qPCR was performed to measure the RNA expression levels.

Cell transfection. LINC00662 shRNA constructs were generated by inserting the LINC00662-targeting shRNA sequences into pENTR/U6 plasmids. The miR497-5p mimics, its inhibitor and corresponding negative controls (NC) were purchased from RiboBio (Guangzhou, China). Transfection of MDA-MB-157 cells was performed by using cell electroporation system operator H1 (Suzhou Etta Biotech Co. Ltd., Suzhou, China.) according to the manufacturer's manual. After transfection, the cells were seeded into appropriate cell culture plates for the further assays.

MTT assay. After transfection, MDA-MB-157 cells were seeded in 96-well plates at $5 \times 10^{3}$ per well, and cultured for the next 48 hours. An MTT assay was performed to measure the cells viability. MTT powder was dissolved in phosphate-buffer saline (PBS) at $10 \mathrm{mg} / \mathrm{mL}$ 
as stock solution, and diluted to $1.0 \mathrm{mg} / \mathrm{ml}$ in the cell medium as working concentration. Briefly, $20 \mu \mathrm{l}$ of MT'T working solution was added to each well and the plates were incubated for 4 hours at $37^{\circ} \mathrm{C}$. Then the medium was removed $200 \mu \mathrm{L}$ of dimethyl sulfoxide (DMSO) was added to each well and the plate was shaken carefully. Finally, the absorbance at $570 \mathrm{~nm}$ was measured for each well using the Microplate Reader (ELX800, BIOTEK Instruments. INC). The effect of shNC on MDAMB-157 cells viability was assessed. Viability of the blank control was expressed as $100 \%$.

Colony formation assay. Cell proliferation of transfected MDA-MB-157 cells was measured with colony formation assay according to the method described by Horibata (Horibata et al., 2015). Briefly, the MDAMB-157 culture medium was replaced with growth medium enriched in $0.3 \%$ agarose. Then the cell suspension was seeded into $0.7 \%$ agarose-precoated- 6 -well cell culture plates. After another 14 days culture, the cells were fixed and stained with $0.1 \%$ crystal violet. Finally, the colonies were counted under a light microscope.

Wound healing assay. To study the migration of MDA-MB-157 cells transfected with different reagents. the wound healing assay was performed as described in (Rodriguez et al., 2005) Briefly, $5 \times 10^{5}$ transfected MDAMB-157 cells were seeded into each well in a 6-well plate. When the cells were cultured to $90 \%$ confluence, a wound was made by a $100 \mu \mathrm{l}$ size pipette tip. After another 48 hours, the wound recovery area was evaluated under a light microscope.

Transwell assay. Transwell assay was performed to determine the invasive capacity of MDA-MB-157 cells according to the method established by Marshall (Marshall 2011). Briefly, $3 \times 10^{4}$ transfected MDA-MB-157 cells were seeded into the upper chamber of Matrigelprecoated transwell and cultured in L-15 medium. The lower chamber contained $10 \%$ FBS growth medium. After 48 hours of culture, the transwelled cells present in the lower chamber were fixed and stained with crystal violet. The pictures were taken under a light microscope. and individual cell colonies were counted.

Luciferase reporter assay. The Dual-Luciferase system was generated by inserting the cDNA fragments containing the putative miR-497-5p binding site from LINC00662 or EglN2 3'-UTR into pmirGLO DualLuciferase miRNA Target Expression Vectors (Promega, Madison, WI, USA). PmirGLO/LINC00662 or pmirGLO/ EglN2 3'-UTR constructs along with miR-497$5 \mathrm{p}$ mimics were co-transfected into MDA-MB-157 cells, then the cells were seeded into a 24 -well plate for about 48 hours culture. Finally, the cells were lysed to measure the Dual-Luciferase Reporters' luciferase activity according to the manufacturer's protocol.

RNA pull-down. Biotin RNA Labeling Mix (Roche Diagnostics, Indianapolis, IN, USA) was used for biotinylating the LINC00662, LINC00662-MUT mimics and NC mimics, which were subsequently transfected into MDA-MB-157 cells. After about 48 hours culture. the cells were harvested to get the lysates. Next, streptavidin-coated magnetic beads were added to the lysates and incubated at $4^{\circ} \mathrm{C}$ for 3 hours. Then the beads were washed and bound RNAs was isolated using RNeasy Mini Kit (Qiagen). Finally, RT-qPCR was performed to measure the abundance of miRNA-497-5p in the bound RNA.

RT-qPCR analysis. Total RNA from the tissue samples and cultured cells was extracted using RNeasy Mini Kit (Qiagen, Dusseldorf, Germany). The miRNA was isolated using miRNeasy Mini kit (Qiagen). RNA was reverse transcribed using High Capacity cDNA Reverse Transcription kit (Applied Biosystems; Thermo Fisher Scientific, Inc.) according to the manufacturer's protocols. PCR amplifications were performed using Power SYBR Green PCR Master Mix (Applied Biosystems) with the synthesized cDNA as the template. Primers of miR-497-5p, U6, EgIN2 and GAPDH were as described in previous studies (Muratsu-Ikeda et al., 2012; Gong et al., 2018; Huang et al., 2019). The PCR primers for miR-497-5p were 5'-CCT'TCAGCAGCACACTGTGG3' (forward) and 5'-CAGTGCAGGGTCCGAGGTAT-3' (reverse); and those for U6 were 5'-CTCGCTTCGGCAGCACA-3' (forward) and 5'-AACGCTTCACGAAT'T'TCGT-3' (reverse). The PCR primers for LINC00662 were 5'-ACACGCT'TCTGAAACTGG-3' (forward) and 5'-GTCAACATGGTGAAACCC-3' (reverse). Primers for EgIN2 were 5'-GGCGATCCCGCCGCGC-3' (forward) And 5'-CCTGGGTAACACGCC -3' (reverse). Primers for GAPDH were 5'-CGAGCCACATCGCTCAGACA-3' (forward) and 5'-GTGGTGAAGACGCCAGTGGA-3' (reverse). The expression levels of EglN2 and LINC00662 were normalized to GAPDH, while miR-497-5p expression was normalized to U6 snRNA. The expression levels were analyzed according to the $2^{-\Delta \Delta c \mathrm{c}}$ method.

Western blot. The cultured cells were harvested in lysis buffer and incubated on ice for $30 \mathrm{~min}$. The supernatant was collected by centrifugation with $15000 \times g$ for $5 \mathrm{~min}$ at $4^{\circ} \mathrm{C}$. Then the proteins $(40 \mu \mathrm{g})$ were separated on $12 \%$ SDS-PAGE and transferred onto PVDF membranes (Millipore) using a MiniGenie blotting system (Bio-Rad). Next, the membranes were blocked for 1 hour with TBST containing $1 \%$ skimmed milk powder and then incubated overnight at $4{ }^{\circ} \mathrm{C}$ with rabbit monoclonal antibodies against human EGLN2/PHD1 (1:1000; cat. no. ab108980; Abcam) or GAPDH (1:1000; cat. no. 5174S; Cell Signaling Technology). Next, the membranes were washed with TBST and incubated with goat-antirabbit secondary antibody (1:10000; cat. no. 14708S; Cell Signaling Technology). Finally, the protein bands on the membranes were visualized with an enhanced chemiluminescence (ECL) system. Densitometric analysis of the bands was done using The ImageJ (National Institutes of Health, Bethesda, MD).

Statistical Analysis. GraphPad Prism (Version 6.01 for Windows) statistical software was used to perform statistical analysis. Student t tests were employed to identify the significant differences between groups. Statistical significance difference was set at $p<0.05$.

\section{RESULTS}

\section{LINC00662 is overexpressed in breast cancer tissues and cell lines}

To confirm whether LINC00662 takes part in breast cancer, we compared its expression level in breast tissues with the expression level in adjacent normal tissues from patients with breast cancer using RT-qPCR. The expression level of LINC00662 in different breast cancer cell lines was detected simultaneously. The results demonstrated that LINC00662 was significantly upregulated in breast cancer tissues compared to the adjacent normal tissue (Fig. 1A). Similarly, LINC00662 level was significantly increased in MDA-MB-157, MDA-MB-468 and MCF-7 cell lines compared with the control CCD1095Sk cell line (Fig. 1B). To explore the subcellular localization of LINC00662 in breast cancer cells, we de- 
A

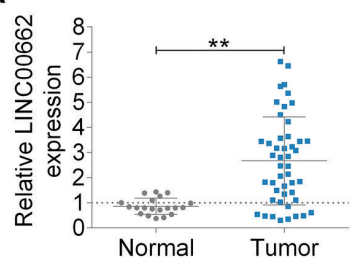

$\mathrm{B}$

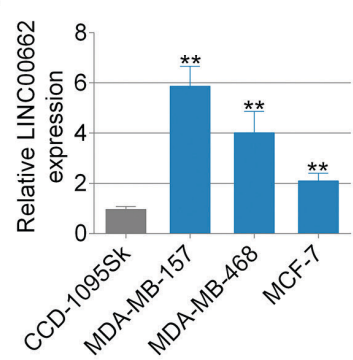

C

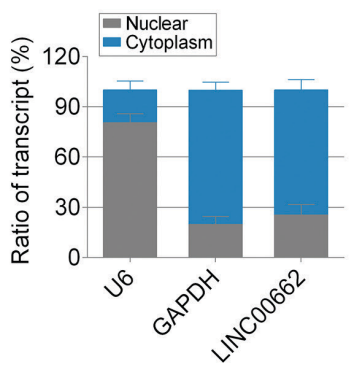

Figure 1. Relative expression of LINC00662 in BC tissues and cell lines.

(A) RT-qPCR analysis was used to determine the relative expression of LINC00662 in breast cancer tissues ( $\mathrm{n}=47$ ) and adjacent normal tissues $(n=20)$. The results were normalized to GAPDH expression. (B) Relative LINC00662 expression in four breast cancer cell lines and one normal human breast fibroblast cell line CCD-1095Sk. (C) After nuclei and cytosol separation, RNA expression levels were measured. GAPDH was used as a cytosol marker and U6 was used as a nucleus marker. Three independent experiments were performed. The graph shows the mean \pm S.D. calculated for at least three experiments. ${ }^{* *} p<0.01$.

termined its level in the cytoplasmic and nuclear RNA fractions of MDA-MB-157 cells. The results showed that LINC00662 was mainly located in the cytoplasm (Fig. 1C). These data confirm that LINC00662 is overexpressed in breast cancer.
LINC00662 promoted the proliferation, migration and invasion of breast cancer cells

Since LINC00662 was upregulated in breast cancer cell lines, we conducted further studies in MDA-MB-157 cells as a cell model to explore biological function of LINC00662 in vitro. MDA-MB-157 cells were transfected
A

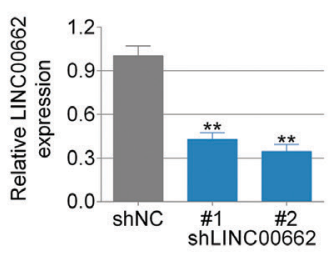

C

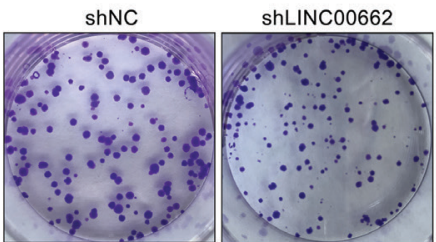

D

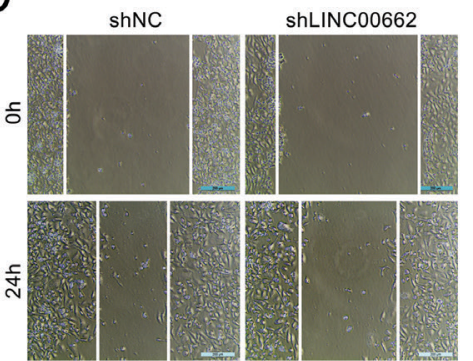

E

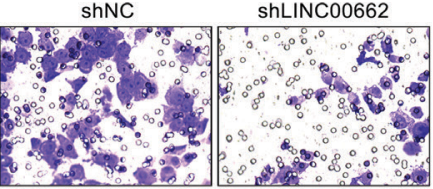

B
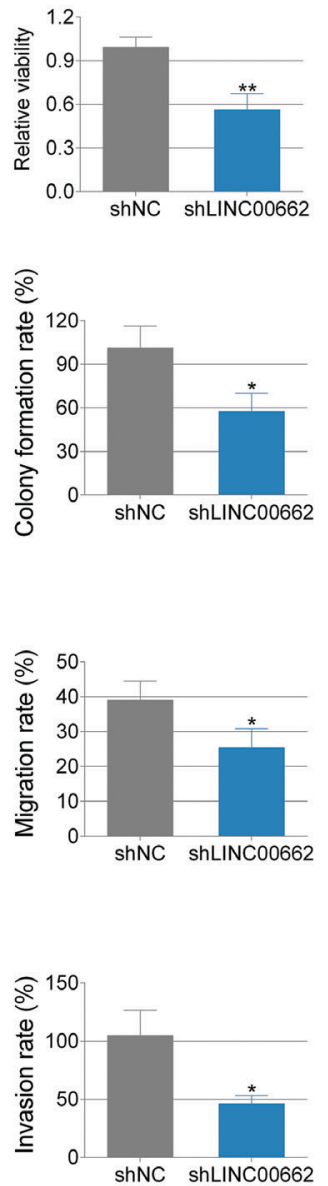

Figure 2. LINC00662 knockdown inhibits proliferation, migration and invasion of MDA-MB-157 cells.

(A) RT-qPCR was used to assess the expression of LINC00662 in MDA-MB-157 cells transfected with different shLINC00662or shNC RNA. MTT assay (B), colony formation assay (C), wound healing assay (D), and transwell assay was (E), were performed to assess the viability, colony formation, migration and invasion of MDA-MB-157 cells transfected with shLINC00662-2\# (hereinafter referred to as shLINC00662) or shNC. Three independent experiments were performed. The graphs show the mean \pm S.D. of at least three experiments. ${ }^{*} p<0.05,{ }^{* *} p<0.01$. 
A

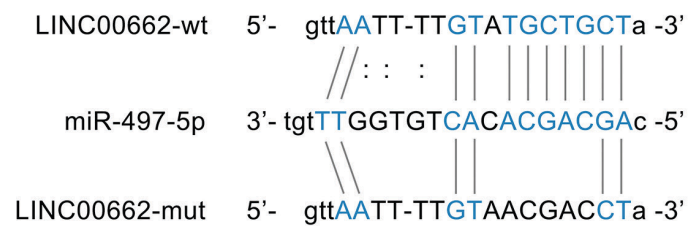

C

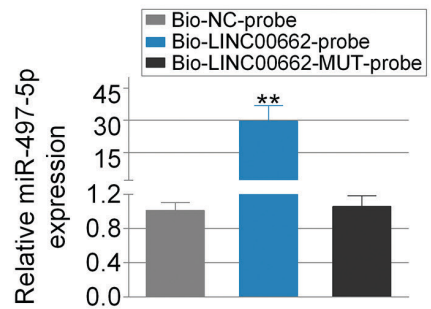

B

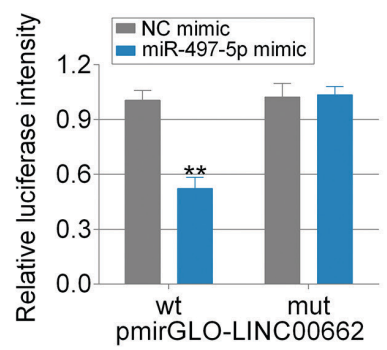

D

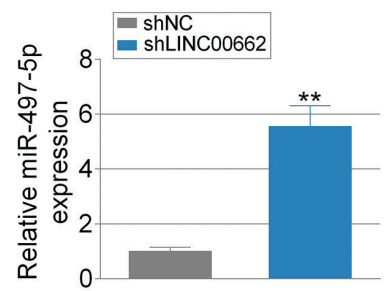

Figure 3. MiR-497-5p is a direct target of LINC00662.

(A) StarBase was employed to predict the target microRNA regulated by LINC00662. Binding region between miR-497-5p and LINC00662 was predicted, and a LINC00662-mut sequences were constructed. (B) The luciferase reporter constructs containing the wild type (LINC00662-wt) or mutant LINC00662 (LINC00662-mut) sequences were co-transfected into MDA-MB-157 cells with miR-497-5p mimic or the corresponding negative control (NC mimic), followed by detecting of the relative luciferase activity. (C) An RNA pull-down assay was performed using biotinylated LINC00662-probe, LINC00662-mut-probe or NC-probe, and the level of miR-497-5p was measured with RTqPCR. (D) RT-qPCR analysis was used to determine the expression of miR497-5p in MDA-MB-157 cells transfected with shLINC00662-2\# or shNC. Three independent experiments were performed. The graph shows the mean \pm S.D. calculated for at least three experiments. ** $p<0.01$.

with shLINC00662-1\#, shLINC00662-2\# or shNC. RTqPCR showed that both shLINC00662 RNAs significantly downregulated the expression of LINC00662 in MDAMB-157 cells, with shLINC00662-2\# (hereinafter referred to as shLINC00662) causing the maximum decrease in LINC00662 expression (Fig. 2A, $p<0.01$ ). MTT assay showed that shLINC00662 remarkedly reduced the viability of MDA-MB-157 cells as compared to shNC (Fig. 2B, $p<0.01)$. Next, a colony formation assay was applied to evaluate the effect of LINC00662 on cell proliferation. As show in Fig. 2C, the decreased expression of LINC00662 led to a significant reduction in MDA-MB-157 cell proliferation $(p<0.05)$. Subsequently, the effect of LINC00662 on cell invasion and migration was examined using wound healing assay and transwell assay. The wound healing assay showed that the reduced level of miR-497-5p remarkably repressed the migratory ability of MDA-MB-157 cells compared to the control (Fig. 2D, $p<0.05$ ). The transwell assay showed that shLINC00662 markedly inhibited the invasion of MDA-MB-157 cells compared to shNC (Fig. $2 \mathrm{E}, p<0.05)$. Taken together, these findings demonstrate that LINC00662 promotes breast cancer cells proliferation, migration and invasion in vitro.

\section{LINC00662 regulated miR-497-5p expression via direct interaction}

Since one way lncRNAs regulates gene expression is to act as a miRNA sponge, we employed bioinformatics analysis to predict the potential miRNAs targets of LINC00662. The result showed one of the potential targets was miR-497-5p (Fig. 3A). Then, dual-luciferase reporter assay was performed to verify this relationship, and the results indicated that miR-497-5p mimic significantly downregulated the luciferase activity of the LINC00662-wt reporter vector(Fig. 3B). In addition, the downregulation of the luciferase activity of LINC00662 reporter vector was abrogated when the predicted binding site was mutated (Fig. 3B). The results of RNA pull-down assay indicated that LINC00662 directly interacted with miR-497-5p in MDA-MB-157 cells (Fig. 3C). Moreover, our results show that LINC00662 knockdown upregulated miR-497-5p expression in MDA-MB-157 cells (Fig. 3D). Overall, these results demonstrated that LINC00662 directly interacted with miR-497-5p and influences its expression in breast cancer cells.

\section{MiR-497-5p regulated EgIN2 expression in MDA-MB-157 cells}

We next explored the target genes of miR-497-5p. Bioinformatics analysis showed that one of the target genes of miR-497-5p was EglN2 (Fig. 4A). We performed a dual-luciferase reporter assay to verify this interaction. The result indicated that the overexpression of miR-497$5 p$ significantly downregulated EgIN2-wt reporter vector's luciferase activity (Fig. 4B, $p<0.01$ ). Meanwhile, overexpression miR-497-5p with a mutation within the predicted LINC00662 binding site did not influence luciferase activity of the EgIN2 reporter vector (Fig. 4B). We determined the expression level of EglN2 in breast cancer tissues and adjacent normal tissues from breast cancer patients with RT-qPCR. The results demonstrated that EgIN2 was significantly upregulated in breast cancer tissues compared to adjacent normal tissues (Fig. 4C). Notably, the expression level of EgIN2 was positively associated with the expression level of LINC00662 in breast tissues (Fig. 4D, $\mathrm{r}=0.6397, p<0.001)$. These data confirmed that miR-497$5 \mathrm{p}$ could regulate EgIN2 expression in breast cancer.

\section{Knockdown of miR497-5p reversed the effect of LINC00662 knockdown in MDA-MB-157 cells}

To confirm the interaction of miR497-5p and LINC00662 in breast cancer, we co-transfected MDA- 
A

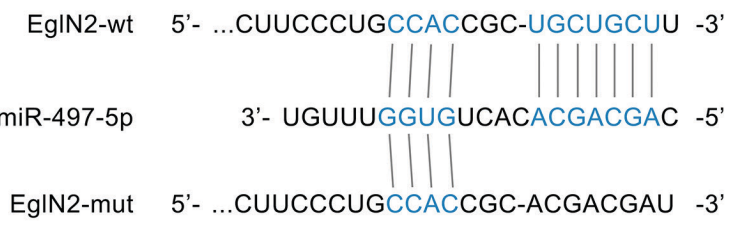

B

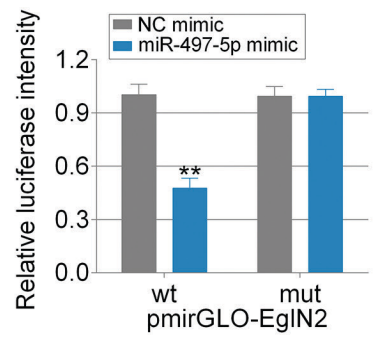

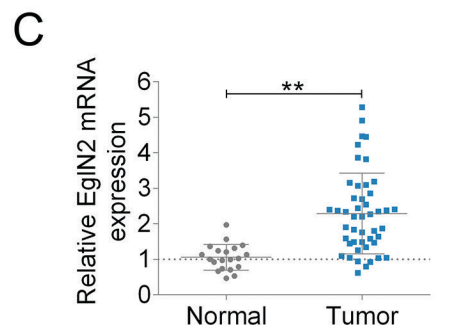

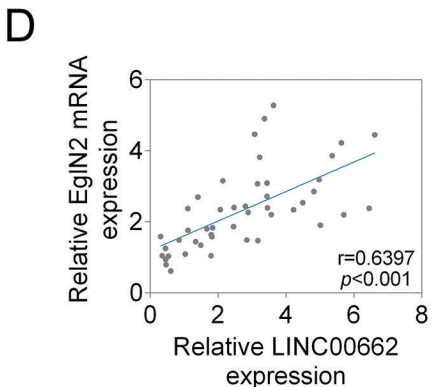

Figure 4. EgIN2 is a target gene of miR-497-5p.

(A) StarBase was employed to predict the target genes regulated by miR49-5p. Binding region between miR-497-5p and EgIN2 was predicted, and an EgIN2-mut sequence was designed. (B) The luciferase reporter constructs containing the wild type (EgIN2-wt) or mutant EgIN2 (EgIN2-mut) sequences were co-transfected into MDA-MB-157 cells with miR-497-5p mimic or the corresponding negative control (NC mimic), followed by the detection of the relative luciferase activity. (C) RT-qPCR analysis was used to detect the expression level of EgIN2 in breast cancer tissues $(n=47)$ and adjacent normal tissues $(n=20)$. The results were normalized to GAPDH expression. (D) The association between the levels of LINC006622 and EgIN2 was analyzed with Spearman's rank correlation test. Three independent experiments were performed. The graph shows the mean \pm S.D. calculated for at least three experiments. ${ }^{* *} p<0.01$.

MB-157 cells with shLINC00662 or shNC along with miR-497-5p inhibitor or its negative control (NC inhibitor). RT-qPCR showed that miR-497-5p was significantly up-regulated in the cells co-transfected with shLINC00662 and NC inhibitor $(p<0.01)$, while miR-497$5 \mathrm{p}$ was significantly down-regulated in the cells co-transfected with shNC and miR-497-5p inhibitor, as compared to the control cells co-transfected with shNC and NC inhibitor $(p<0.01)$. However, co-transfection of shLINC00662 along with miR-497-5p inhibitor remarkedly lowered the expression of miR-497-5p compared to the cells co-transfected with shLINC00662 and NC inhibitor $(p<0.01)$, but still, miR-497-5p expression level was similar to the control cells co-transfected with shNC and NC inhibitor (Fig. 5A). MTT assay showed that transfection of shLINC00662 along with miR-497-5p inhibitor markedly improved the viability of MDA-MB-157 cells compared with the shLINC00662 and NC inhibitor cotransfection (Fig. 5B, $p<0.05$ ). A colony formation assay was applied to further evaluate the effect of miR-497-5p on cell proliferation. As shown in Fig. 5C, downregulation of miR-497-5p led to a significant increase in the proliferation of the MDA-MB-157 cells with LINC00662 knockdown $(p<0.05)$. Furthermore, the wound healing assay showed that downregulation of miR-497-5p remarkably improved the migration ability of the MDAMB-157 cells with LINC00662 knockdown, as compared to the cells co-transfected with shLINC00662 and NC inhibitor (Fig. 5D, $p<0.05$ ). Similarly, the transwell assay showed that the downregulation of miR-497-5p markedly improved the invasion of MDA-MB-157 cells with LINC00662 knockdown (Fig. 5E, $p<0.05$ ). Western blot showed that LINC00662 knockdown was enough to decrease the expression level of EgIN2 in the MDAMB-157 cells. However, the expression level of EglN2 was upregulated by miR-497-5p inhibitor, as the level of EglN2 in the miR-497-5p knockdown cells both cotransfected with shLINC00662 or shNC was significantly higher than the miR-497-5p negative control cells cotransfected with shLINC00662 (Fig. 5F, $p<0.05$ ). Taken together, these results demonstrated that the knockdown of miR497-5p reversed the effect of LINC00662 knockdown in MDA-MB-157 cells.

\section{DISCUSSION}

Breast cancer is one of the most common and aggressive malignancies among women around the word. Both the incidence and mortality from breast cancer rank first among all cancers in women across 20 world regions (Bray et al., 2018). To reduce the harm of breast cancer to women, prevention strategies such as weight control, high-quality screening and diagnosis have been suggested (Zeng et al., 2014). As it is a multifactorial disease, many molecular changes associated with this cancer have been extensively studied in the past (Bertoli et al., 2015; Lou et al., 2018; Leithner et al., 2019; Pasha et al., 2019). In the present research, we demonstrated that LINC00662 was up-regulated in breast cancer tissues and cell lines. Knockdown of LINC00662 dramatically inhibited the proliferation, migration and invasion of MDA-MB-157 cells in vitro. This preliminary research indicated that LINC00662 might be a promising candidate in breast cancer diagnosis and therapy.

Increasing evidence indicates that dysregulation of lncRNAs plays an important role in tumorigenesis and metastasis in human cancer (Han et al., 2019; Sun et al., 2019). In breast cancer, several lncRNAs have been identified as indicators for patients' prognosis. For ex- 
A
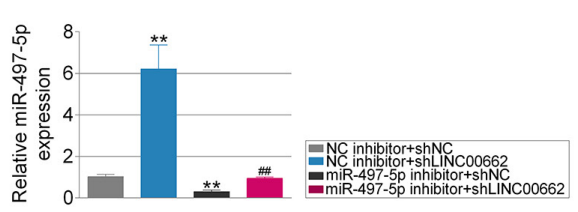

C

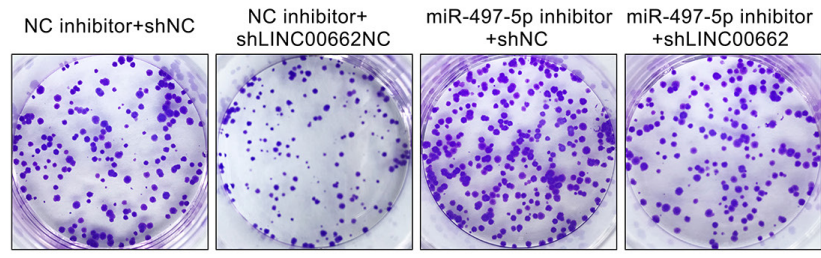

D

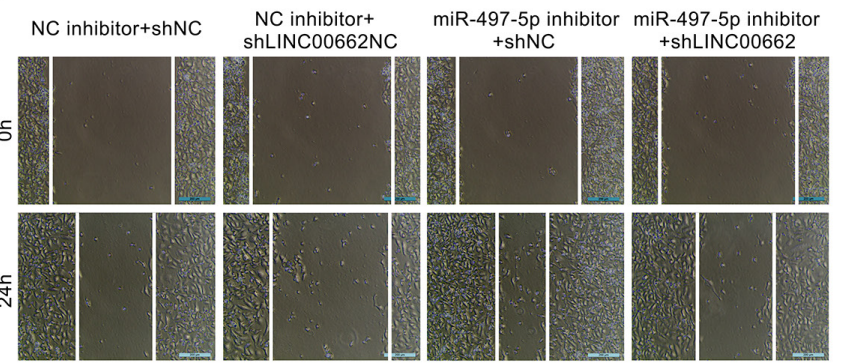

E

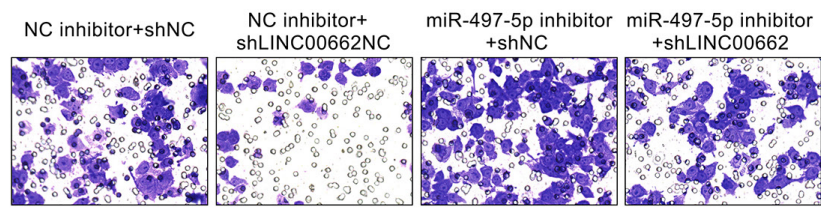

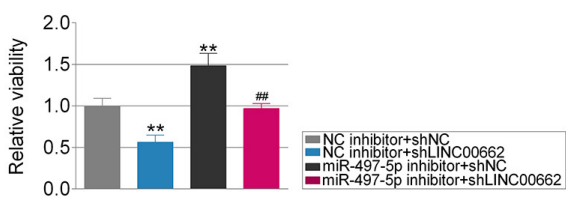
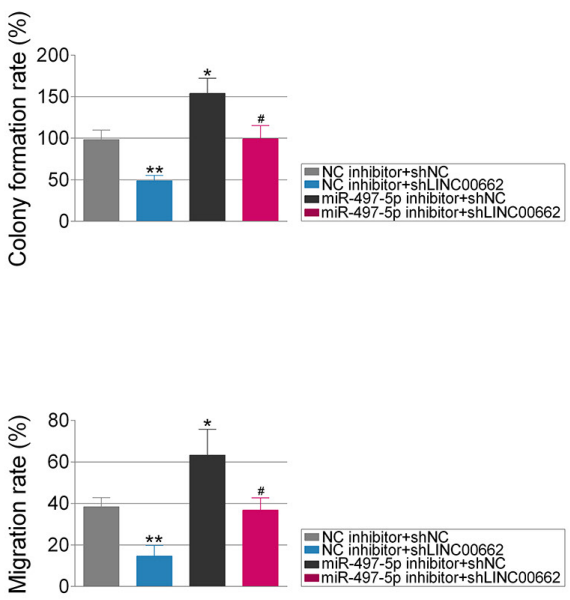

$\mathrm{F}$
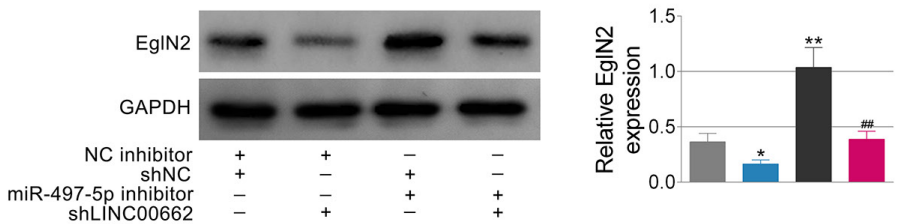

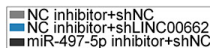

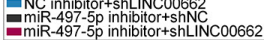

Figure 5. Knockdown of miR497-5p reversed the effect of LINC00662 knockdown in MDA-MB-157 cells.

shLINC00662 or shNC along with miR-497-5p inhibitor or NC inhibitor were co-transfected into MDA-MB-157 cells. After transfection, the cells were seeded into appropriate cell culture plates for further assays. (A) RT-qPCR was used to assess the expression of miR497-5p. MTT assay (B), colony formation assay (C), wound healing assay (D), and transwell assay (E) were performed to assess the viability, colony formation, migration and invasion of the MDA-MB-157 cells. (F) Western blot analysis was performed to measure the expression level of EgIN2 in the MDA-MB-157 cells. Three independent experiments were performed. The graph shows the mean \pm S.D. calculated for at least three experiments. ${ }^{*} p<0.05,{ }^{* *} p<0.01$.

amples, lncRNA AP000924.1, AC091043.1 and FOXCUT were reported to have strong diagnostic values for predicting the existence of human triple-negative breast cancer (TNBC), as they were upregulated in TNBC (Fan et al., 2019). Circulating long noncoding RNA GAS5 was reported to have the potential to be used for assessing the surgical effects and prognosis for breast cancer patients (Han et al., 2016). In the present research, we demonstrated that LINC00662 expression level was significantly higher in breast cancer tissues and cell lines (MDA-MB-157, MDA-MB-468 and MCF-7) compared to the adjacent normal tissue and human breast fibroblast cell line (CCD-1095Sk).

LINC00662 was first reported in lung squamous cell carcinoma (Liu et al., 2017). After that, the role of LINC00662 was studied in several other tumors. In lung cancer H1299 and A549 cell lines, LINC00662 could influence cell invasion, metastasis and cancer stem cell-like phenotypes, (Gong et al., 2018). In gastric cancer cells, the knockdown of LINC00662 increased the chemosensitivity and decreased GC cell proliferation (Liu et al., 2018). Our study demonstrated that LINC00662 knockdown could inhibit the proliferation, migration and invasion of breast cancer cells (MDA-MB-157).

Increasing evidence suggests that lncRNAs could play a role of miRNA sponges to downregulate the activity of target miRNAs in many cancers (Li et al., 2019; Liao et al., 2019). LINC00662 has been reported to serve as a microRNA-340-5p sponge in acute myeloid leukemia cells (Liu et al., 2019). Here, bioinformatic analysis identified miR497-5p as the target miRNAs of LINC00662, as they have complementary binding sites.. The binding 
sites of miR-497-5p were also found in the 3'-UTR of EglN2, and they were validated with the luciferase assay.

A previous study has shown that miR-497-5p was downregulated in human non-small-cell lung cancer tissues and cell lines, and indicated that miR-497-5p could inhibit tumor cell growth and invasion by inhibiting the expression of SRY-box transcription factor 5 (Li et al., 2019). Similarly, miR-497-5p was remarkably downregulated in the clinical cancer samples as well as in colorectal cancer cell lines, and it targets protein tyrosine phosphatase non-receptor type 3 (PTPN3) to inhibit cell proliferation and migration (Hong et al., 2019). Most importantly, an independent study has shown that miR-497 was downregulated in breast cancer cells (Li et al., 2019). As a prolyl hydroxylase, EGLN2 participates in suppression of mammary gland proliferation in vivo (Zhang et al., 2009). Besides that, EglN2 could positively regulate mitochondrial function through interaction with NRF1 and PGC1alpha in breast cancer (Zhu et al., 2016). With the regulation of long non-coding RNA NORAD- MIR-205 axis, EglN2 could promote invasion and migration in malignant melanoma (Chen et al., 2019). We found that LINC00662 can downregulate the expression of miR497-5p via direct interaction and upregulate the expression of EglN2 in MDA-MB-157 cells. At the same time, we found that miR-497-5p decreased the expression of EglN2 and reversed the effect of LINC00662 knockdown in MDA-MB-157 cells.

In summary, the present study provides evidence that LINC00662 modulates miR-497-5p / EgIN2 pathway to facilitate breast cancer progression. Our data suggests that LINC00662- miR-497-5p - EgIN2 signal axis may provide a new therapeutic target for breast cancer prevention.

\section{Acknowledgements}

Not applicable.

\section{Competing interests}

The authors state that there are no conflicts of interest to disclose.

\section{Ethics approval and consent to participate}

The present study was approved by the Ethics Committee of The First Affiliated Hospital of Wannan Medical College, and all the patients signed written informed consent.

\section{Availability of data and materials}

All data generated or analyzed during this study are included in this published article.

\section{Authors' contributions}

LC and ZHX conceived and designed the experiments- PZ analyzed and interpreted the results of the experiments, WQX performed the experiments

\section{Patient consent for publication}

Not Applicable

\section{REFERENCES}

Bertoli G, Cava C, Castiglioni I (2015) MicroRNAs: new biomarkers for diagnosis, prognosis, therapy prediction and therapeutic tools for breast cancer. Theranostics 5: 1122-1143. https://doi. org/10.7150/thno. 11543
Bray F, Ferlay J, Soerjomataram I, Siegel RL, Torre LA, Jemal A (2018) Global cancer statistics 2018: GLOBOCAN estimates of incidence and mortality worldwide for 36 cancers in 185 countries. CA Cancer J Clin. 68: 394-424. https://doi.org/10.3322/caac.21492

Chen W, Zheng R, Baade PD, Zhang S, Zeng H, Bray F, Jemal A, Yu $\mathrm{XO}, \mathrm{He} \mathrm{J}$ (2016) Cancer statistics in China, 2015. CA Cancer J. Clin. 66: 115-132. https://doi.org/10.3322/ caac.21338

Chen Y, Cao K, Li J, Wang A, Sun L, Tang J, Xiong W, Zhou X, Chen X, Zhou J, Liu Y (2019) Overexpression of long non-coding RNA NORAD promotes invasion and migration in malignant melanoma via regulating the MIR-205-EGLN2 pathway. Cancer Med. 8: 1744-1754. https://doi.org/10.1002/cam4.2046

DiStefano JK (2018) The emerging role of long noncoding RNAs in human disease. Methods Mol. Biol. 1706: 91-110. https://doi. org/10.1007/978-1-4939-7471-9 6

Fan CN, Ma L, Liu N (2019) Comprehensive analysis of novel threelong noncoding RNA signatures as a diagnostic and prognostic biomarkers of human triple-negative breast cancer. I Cell. Biochem. 120: 3185-3196. https://doi.org/10.1002/jcb.27584

Fasih A, Fonge H, Cai, Leyton JV, Tikhomirov I, Done SJ, Reilly RM (2012) (1)(1)(1)In-Bn-DTPA-nimotuzumab with/without modification with nuclear translocation sequence (NLS) peptides: an Auger electron-emitting radioimmunotherapeutic agent for EGFR-positive and trastuzumab (Herceptin)-resistant breast cancer. Breast Cancer Res. Treat. 135: 189-200. https://doi.org/10.1007/s10549-0122137-y

Gong W, Su Y, Liu Y, Sun P, Wang X (2018) Long non-coding RNA Linc00662 promotes cell invasion and contributes to cancer stem cell-like phenotypes in lung cancer cells. J. Biochem. 164: 461-469. https://doi.org/10.1093/jb/mvy078

Han L, Ma P, Liu SM, Zhou X (2016) Circulating long noncoding RNA GAS5 as a potential biomarker in breast cancer for assessing the surgical effects. Tumour Biol. 37: 6847-6854. https://doi. org/10.1007/s13277-015-4568-7

Han S, Jin X, Liu Z, Xing F, Han Y, Yu X, G He G, Qiu F (2019) The long non-coding RNA HOTTIP promotes breast cancer cell migration, invasion, and epithelial-mesenchymal transition via Wnt/ beta-catenin pathway. Biochem. Cell. Biol. 97: 655-664. https://doi. org/10.1139/bcb-2018-0313

Hong S, Yan Z, Wang H, Din L, Bi M(2019) Upregulation of microRNA-497-5p inhibits colorectal cancer cell proliferation and invasion via targeting PTPN3. Biosci. Rep. 39: BSR20191123. https://doi. org/10.1042/BSR20191123

Horibata S, Vo TV, Subramanian V, Thompson PR, Coonrod SA (2015) Utilization of the soft agar colony formation assay to identify inhibitors of tumorigenicity in breast cancer cells. J. Vis. Exp. e52727. https://doi.org/10.3791/52727

Huang X, Wang L, Liu W, Li F (2019) MicroRNA-497-5p inhibits proliferation and invasion of non-small cell lung cancer by regulating FGF2. Oncol. Lett. 17: 3425-3431. https://doi.org/10.3892/ ol.2019.9954

Lalevee S, Feil R (2015) Long noncoding RNAs in human disease: emerging mechanisms and therapeutic strategies. Epigenomics 7: 877879. https://doi.org/10.2217/epi.15.55

Leithner D, Horvat JV, Bernard-Davila B, Helbich TH, OchoaAlbiztegui RE, Martinez DF, Zhang M, Thakur SB, Wengert GJ, Staudenherz A, Jochelson MS, Morris EA, Baltzer P'TA, Clauser P, Kapetas P, Pinker K (2019) A multiparametric [(18)F]FDG PET/ MRI diagnostic model including imaging biomarkers of the tumor and contralateral healthy breast tissue aids breast cancer diagnosis. Eur. J. Nucleic Med. Mol. Imaging 46: 1878-1888. https://doi. org/10.1007/s00259-019-04331-6

Li G, Wang K, Wang J, Qin S, Sun X, Ren H (2019) miR-497-5p inhibits tumor cell growth and invasion by targeting SOX5 in nonsmall-cell lung cancer. J. Cell. Biochem. 120: 10587-10595. https:// doi.org/10.1002/jcb.28345

Li L, Ji J, Wang JB, Niyazi M, YL Qiao YL, Boffetta P (2012) Attributable causes of breast cancer and ovarian cancer in china: reproductive factors, oral contraceptives and hormone replacement therapy. Chin. J. Cancer Res. 24: 9-17. https://doi.org/10.1007/ s11670-012-0009-y

Li N, Zhang LY, Qiao YH, Song RJ (2019) Long noncoding RNA LINC00662 functions as miRNA sponge to promote the prostate cancer tumorigenesis through targeting miR-34a. Eur. Rev. Med. Pharmacol. Sci. 23: 3688-3698. https://doi.org/10.26355/eurrev_201905_17792

Li X, Wang Q, Rui Y, Zhang C, Wang W, Gu J, Tang J, Ding Y (2019) HOXC13-AS promotes breast cancer cell growth through regulating miR-497-5p/PTEN axis. J. Cell. Physiol. 234: 22343-22351. https:// doi.org/10.1002/jcp. 28800

Li XY, Zhou LY, Luo H, Zhu Q, Zuo L, Liu GY, Feng C, Zhao JY, Zhang YY, Li X (2019) The long noncoding RNA MIR210HG promotes tumor metastasis by acting as a ceRNA of miR-1226-3p to regulate mucin-1c expression in invasive breast cancer. Aging (Albany NY) 11: 5646-5665. https://doi.org/10.18632/aging.102149 
Liao S, Xing S, Ma Y (2019) LncRNA SNHG16 sponges miR-98-5p to regulate cellular processes in osteosarcoma. Cancer Chemother. Pharmacol. 83: 1065-1074. https://doi.org/10.1007/s00280-019-03822-5

Liu B, Y Chen Y, Yang J (2017) LncRNAs are altered in lung squamous cell carcinoma and lung adenocarcinoma. Oncotarget 8: 2427524291. https://doi.org/10.18632/oncotarget.13651

Liu Y, Gao X, Tian X (2019) High expression of long intergenic non-coding RNA LINC00662 contributes to malignant growth of acute myeloid leukemia cells by upregulating ROCK1 via sponging microRNA-340-5p. Eur. J. Pharmacol. 859: 172535. https://doi. org/10.1016/j.ejphar.2019.172535

Liu Z, Yao Y, Huang S, Li Li, Jiang B, Guo H, Lei W, Xiong J, Deng J (2018) LINC00662 promotes gastric cancer cell growth by modulating the Hippo-YAP1 pathway. Biochem. Biophys. Res. Commun. 505: 843-849. https://doi.org/10.1016/j.bbrc.2018.09.191

Lou KX, Li ZH, Wang P, Liu Z, Chen Y, Wang XL, HX Cui HX (2018) Long non-coding RNA BANCR indicates poor prognosis for breast cancer and promotes cell proliferation and invasion. Eur Rev. Med. Pharmacol. Sci. 22: 1358-1365. https://doi.org/10.26355/ eurrev_201803_14479

Lv P, Qiu X, Gu Y, Yang X, Xu X, Yang Y (2019) Long non-coding RNA SNHG6 enhances cell proliferation, migration and invasion by regulating miR-26a-5p/MAPK6 in breast cancer. Biomed. Pharmacother. 110: 294-301. https://doi.org/10.1016/j.biopha.2018.11.016

Marshall J (2011) Transwell((R)) invasion assays. Methods Mol. Biol. 769. 97-110. https://doi.org/10.1007/978-1-61779-207-6_8

Muratsu-Ikeda S, Nangaku M, Ikeda Y, Tanaka T, Wada T, Inagi R (2012) Downregulation of miR-205 modulates cell susceptibility to oxidative and endoplasmic reticulum stresses in renal tubular cells. PLoS One 7: e41462. https://doi.org/10.1371/journal.pone.0041462
Pasha HA, Rezk NA, Riad MA (2019) Circulating cell free nuclear DNA, mitochondrial DNA and global DNA methylation: potential noninvasive biomarkers for breast cancer diagnosis. Cancer Invest. 1-8. https://doi.org/10.1080/07357907.2019.1663864

Qiu MT, Hu JW, Yin R, Xu L (2013) Long noncoding RNA: an emerging paradigm of cancer research. Tumour Biol. 34: 613-620. https://doi.org/10.1007/s13277-013-0658-6

Rodriguez LG, Wu X, Guan JL (2005) Wound-healing assay. Methods Mol. Biol. 294: 23-29. https://doi.org/10.1385/1-59259-860-9:023

Sun X, Huang T, Zhang C, Zhang S, Wang Y, Zhang Q, Z Liu Z (2019) Long non-coding RNA LINC00968 reduces cell proliferation and migration and angiogenesis in breast cancer through upregulation of PROX1 by reducing hsa-miR-423-5p. Cell. Cycle 18: 1908-1924. https://doi.org/10.1080/15384101.2019.1632641

Xue M, Zhuo Y, Shan B (2017) MicroRNAs, long noncoding RNAs, and their functions in human disease. Methods Mol. Biol. 1617: 1-25. https://doi.org/10.1007/978-1-4939-7046-9_1

Zeng H, Zheng R, Zhang S, Zou X, Chen W (2014) Female breast cancer statistics of 2010 in China: estimates based on data from 145 population-based cancer registries. J. Thorac. Dis. 6: 466-470. https://doi.org/10.3978/j.issn.2072-1439.2014.03.03

Zhang Q, Gu J, Li L, Liu J, Luo B, Cheung HW, Boehm JS, Ni M, Geisen C, Root DE, Polyak K, Brown M, AL Richardson AL, Hahn WC, Kaelin WG Jr, Bommi-Reddy A (2009) Control of cyclin D1 and breast tumorigenesis by the EglN2 prolyl hydroxylase. Cancer Cell. 16: 413-424. https://doi.org/10.1016/j.ccr.2009.09.029

Zhu D-Y, Li X-N, Qi Y, Liu D-L, Yang Y, Zhao J, Zhang C-Y, Wu $\mathrm{K}$, Zhao S (2016) MiR-454 promotes the progression of human non-small cell lung cancer and directly targets PTEN. Biomed. Pharmacother. 81: 79-85. 